\title{
Semiclassical solution to the BFKL equation with massive gluons
}

\author{
Eugene Levin ${ }^{1,3}$, Lev Lipatov², Marat Siddikov ${ }^{3, a}$ \\ ${ }_{1}^{1}$ Department of Particle Physics, School of Physics and Astronomy, Tel Aviv University, Tel Aviv 69978, Israel \\ 2 Theoretical Physics Department, Petersburg Nuclear Physics Institute, Orlova Roscha, Gatchina, 188300 St. Petersburg, Russia \\ ${ }^{3}$ Departamento de Física, Universidad Técnica Federico Santa María and Centro Científico-Tecnológico de Valparaíso, Casilla 110-V, \\ Valparaiso, Chile
}

Received: 24 August 2015 / Accepted: 5 November 2015 / Published online: 26 November 2015

(c) The Author(s) 2015. This article is published with open access at Springerlink.com

\begin{abstract}
In this paper we proceed to study the high energy behavior of scattering amplitudes in a simple field model, with the Higgs mechanism for the gauge boson mass. The spectrum of the $j$-plane singularities of the $t$-channel partial waves and the corresponding eigenfunctions of the BFKL equation in leading $\log (1 / x)$ approximation were previously calculated numerically. Here we develop a semiclassical approach to investigate the influence of the exponential decrease of the impact parameter dependence existing in this model, on the high energy asymptotic behavior of the scattering amplitude. This approach is much simpler than our earlier numerical calculations, and it reproduces those results. The analytical (semi-analytical) solutions which have been found in the approximation can be used to incorporate correctly the large impact parameter behavior in the framework of CGC/saturation approach. This behavior is interesting as it provides the high energy amplitude for the electroweak theory, which can be measured experimentally.
\end{abstract}

\section{Introduction}

In [1] we solved the BFKL equation with a massive gluon in the framework of the Higgs model numerically. Such an equation arises in the electroweak theory with zero Weinberg angle (see Ref. [2]). From a theoretical point of view this model is an instructive example of the gauge invariant theory in which the scattering amplitude has the correct large impact parameter behavior (scattering amplitude $\propto \exp (-m b)$ at large $b$ ) but still has the unitarity problem as the scattering amplitude increases as $s^{\Delta}$ at high energies. Therefore, this model is a perfect training ground to study how the correct $b$ behavior can influence the resolution of the unitarity problem in the framework of the CGC/saturation approach [3-9]. This approach leads to a partial amplitude smaller than unity,

a e-mail: Marat.Siddikov@usm.cl as required by unitarity constraints. However, it generates a radius of interaction that increases with a power of the energy [10-13], leading to a violation of the Froissart bound [14,15].

Another facet of this model is that it is a possible candidate for an effective theory equivalent to perturbative QCD, in the region of distances $(r)$ shorter than $1 / m$, where $m$ denotes the gluon mass. Indeed, for $r \gtrsim 1 / m$ similar correlation functions arise from fixing or eliminating Gribov's copies [16] (see Refs. [17-21]). We wish to emphasize that a gauge theory with the Higgs mechanism leads to a good description of the gluon propagator, calculated in a lattice approach [22,23] with $m=0.54 \mathrm{GeV}$. Therefore, a plausible scenario is that the Higgs gauge theory describes QCD in the kinematic region $r \leq 1 / m$, while for $r \sim 1 / \Lambda_{\mathrm{QCD}}>1 / m$ the non-perturbative QCD approach takes over, and it leads to the confinement of quarks and gluons, which is missing in the theory with a massive gluon.

We found in [1] that the spectrum of the massive BFKL equation in $\omega$-space for $t=0$ is the same as in the massless case [24-28]. The simple parametrizations of the eigenfunctions have also been obtained in Ref. [1]. In this paper we propose using the solution to the BFKL equation with massive gluons, which has the advantage of being simple and semianalytic. Having this solution in hand, we are able to progress to more difficult problems, e.g. a generalization of the main equations of the CGC/saturation approach [9,29-42].

The equation for the scattering amplitude in leading $\ln (1 / x)$ approximation of perturbative theory has been on the market for some time [24,25]; it is schematically shown in Fig. 1. Its kernel is of the form $[1,24,25]$

$$
\begin{aligned}
K\left(q_{1}, q_{2} \mid q_{1}^{\prime}, q_{2}^{\prime}\right)= & \frac{\alpha_{S} N_{c}}{2 \pi^{2}}\left\{\frac{1}{k^{2}+m^{2}}\left(\frac{q_{1}^{2}+m^{2}}{q_{1}^{\prime 2}+m^{2}}+\frac{q_{2}^{2}+m^{2}}{q_{2}^{\prime 2}+m^{2}}\right)\right. \\
& \left.-\frac{q^{2}+\frac{N_{c}^{2}+1}{N_{c}^{2}} m^{2}}{\left(q_{1}^{\prime 2}+m^{2}\right)\left(q_{2}^{\prime 2}+m^{2}\right)}\right\}
\end{aligned}
$$




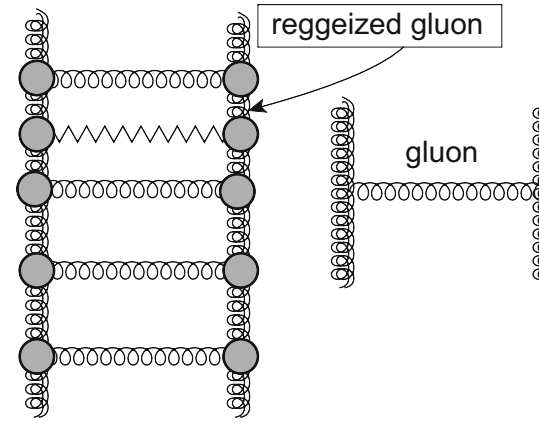

(a)

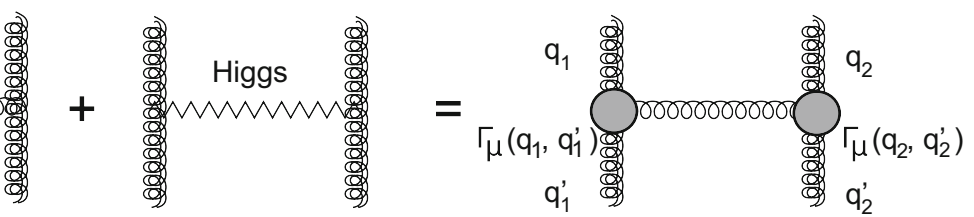

(b)

Fig. 1 The massive BFKL equation (a) and its kernel (b)

For $q=0$ the kernel (1) simplifies considerably and yields a homogeneous BFKL equation for the Yang-Mills theory with the Higgs mechanism,

$$
\begin{aligned}
\omega f(p)= & 2 \omega(p) f(p)+\frac{\alpha_{S} N_{c}}{2 \pi^{2}} \int \mathrm{d}^{2} p^{\prime} \\
& \times\left(\frac{2 f\left(p^{\prime}\right)}{\left(\mathbf{p}-\mathbf{p}^{\prime}\right)^{2}+m^{2}}-\frac{\frac{N_{c}^{2}+1}{N_{c}^{2}} m^{2} f\left(p^{\prime}\right)}{\left(p^{2}+m^{2}\right)\left(p^{\prime 2}+m^{2}\right)}\right),
\end{aligned}
$$

where $q_{1}=q_{2}=p, q_{1}^{\prime}=q_{2}^{\prime}=p^{\prime}$, and $\omega(p)$ is the gluon Regge trajectory given by

$$
\begin{aligned}
\omega(p) & =-\frac{\alpha_{S} N_{c}}{4 \pi^{2}} \int \frac{\mathrm{d}^{2} k\left(p^{2}+m^{2}\right)}{\left(k^{2}+m^{2}\right)\left((\mathbf{p}-\mathbf{k})^{2}+m^{2}\right)} \\
& =-\frac{\alpha_{S} N_{c}}{2 \pi^{2}} \frac{p^{2}+m^{2}}{|p| \sqrt{p^{2}+4 m^{2}}} \ln \frac{\sqrt{p^{2}+4 m^{2}}+|p|}{\sqrt{p^{2}+4 m^{2}}-|p|} .
\end{aligned}
$$

Examining the rotationally symmetric solution, the kernel can be integrated over the azimuthal angle $\phi$. Introducing the new variables

$\kappa=\frac{p^{2}}{m^{2}} ; \quad \kappa^{\prime}=\frac{p^{\prime 2}}{m^{2}} ; \quad E=-\frac{\omega}{\bar{\alpha}_{S}} ; \quad \bar{\alpha}_{S}=\frac{\alpha_{S} N_{c}}{\pi}$,

and changing the notation of the wave function $f(p)$ to $\phi_{E}(\kappa)$, we obtain the one-dimensional BFKL equation

$$
\begin{aligned}
E \phi_{E}(\kappa)= & T(\kappa) \phi_{E}(\kappa)-\int_{0}^{\infty} \frac{\mathrm{d} \kappa^{\prime} \phi_{E}\left(\kappa^{\prime}\right)}{\sqrt{\left(\kappa-\kappa^{\prime}\right)^{2}+2\left(\kappa+\kappa^{\prime}\right)+1}} \\
& +\frac{N_{c}^{2}+1}{2 N_{c}^{2}} \frac{1}{\kappa+1} \int_{0}^{\infty} \frac{\phi_{E}\left(\kappa^{\prime}\right) \mathrm{d} \kappa^{\prime}}{\kappa^{\prime}+1}
\end{aligned}
$$

where the kinetic energy is given by

$T(\kappa)=\frac{\kappa+1}{\sqrt{\kappa} \sqrt{\kappa+4}} \ln \frac{\sqrt{\kappa+4}+\sqrt{\kappa}}{\sqrt{\kappa+4}-\sqrt{\kappa}}$.
In this paper we will work mostly with the Fourierconjugate wave function in the $Y$-representation,

$\Psi(Y, \kappa)=\int_{\epsilon-i \infty}^{\epsilon+i \infty} \frac{\mathrm{d} E}{2 \pi i} e^{-E Y} \phi_{E}(\kappa)$,

for which Eq. (5) takes the form

$$
\begin{aligned}
\frac{\partial \Psi(Y, \kappa)}{\partial Y}= & -T(\kappa) \Psi(Y, \kappa) \\
& +\int_{0}^{\infty} \frac{\mathrm{d} \kappa^{\prime} \Psi\left(Y, \kappa^{\prime}\right)}{\sqrt{\left(\kappa-\kappa^{\prime}\right)^{2}+2\left(\kappa+\kappa^{\prime}\right)+1}} \\
& -\frac{N_{c}^{2}+1}{2 N_{c}^{2}} \frac{1}{\kappa+1} \int_{0}^{\infty} \frac{\Psi\left(Y, \kappa^{\prime}\right) \mathrm{d} \kappa^{\prime}}{\kappa^{\prime}+1} .
\end{aligned}
$$

For completeness of presentation, we recall that the massless BFKL equations have the following form:

$$
\begin{aligned}
E \phi_{E}^{\mathrm{BFKL}}(\kappa) \underset{\epsilon \rightarrow 0}{=} \ln \left(\frac{\kappa}{\epsilon}\right) \phi_{E}^{\mathrm{BFKL}} \\
-\int_{0}^{\infty} \frac{\mathrm{d} \kappa^{\prime} \phi_{E}^{\mathrm{BFKL}}\left(\kappa^{\prime}\right)}{\left|\kappa-\kappa^{\prime}\right|+\sqrt{\kappa \epsilon}} \\
=-\int_{0}^{\infty} \frac{\mathrm{d} \kappa^{\prime}\left(\phi_{E}^{\mathrm{BFKL}}\left(\kappa^{\prime}\right)-\phi_{E}^{\mathrm{BFKL}}(\kappa)\right)}{\left|\kappa-\kappa^{\prime}\right|}
\end{aligned}
$$

$$
\begin{aligned}
\frac{\partial \Psi^{\mathrm{BFKL}}(Y, \kappa)}{\partial Y} \underset{\epsilon \rightarrow 0}{=} & -\ln \left(\frac{\kappa}{\epsilon}\right) \Psi^{\mathrm{BFKL}}(Y, \kappa) \\
& +\int_{0}^{\infty} \frac{\mathrm{d} \kappa^{\prime} \Psi^{\mathrm{BFKL}}\left(Y, \kappa^{\prime}\right)}{\left|\kappa-\kappa^{\prime}\right|+\sqrt{\kappa \epsilon}} .
\end{aligned}
$$

The way of regularization at $\epsilon \rightarrow 0$ stems directly from Eq. (2), considering small masses $\epsilon \mathrm{m}^{2}$ instead of $m^{2}$. Then Eq. (10) can be rewritten with a different way of regularization, for example 


$$
\begin{aligned}
E \phi_{E}^{\mathrm{BFKL}}(\kappa) \underset{\epsilon \rightarrow 0}{=} & -\ln \left(\frac{\epsilon^{2}}{2}\right) \phi_{E}^{\mathrm{BFKL}} \\
& -\int_{0}^{\infty} \mathrm{d} \kappa^{\prime} \phi_{E}^{\mathrm{BFKL}}\left(\kappa^{\prime}\right) \frac{\Theta\left(\left|\kappa-\kappa^{\prime}\right|-\epsilon\right)}{\left|\kappa-\kappa^{\prime}\right|+\epsilon},
\end{aligned}
$$

where $\Theta(z)$ is the unit step function which is equal 1 for $z>0$ and 0 for $z<0$. At large $\kappa$, the solutions of Eq. (5) should coincide with the eigenfunctions of Eq. (9), which are well known [24-28] and have the form

$$
\begin{aligned}
& \phi_{E}(\kappa) \stackrel{\kappa \rightarrow \infty}{\longrightarrow} \phi_{E}^{\mathrm{BFKL}}(\kappa) \sim \kappa^{\gamma-1} \text { with } \\
& E(\gamma)=-\chi(\gamma)=\psi(\gamma)+\psi(1-\gamma)-2 \psi(1),
\end{aligned}
$$

where $\psi(x)=\Gamma^{\prime}(x) / \Gamma(x)$ is a digamma function. Two eigenfunctions, $\phi_{E}(\kappa) \propto \kappa^{\gamma-1}$ and $\phi_{E}(\kappa) \propto \kappa^{-\gamma}$, describe the states with the same energy. For $\gamma=\frac{1}{2}+i v$ these eigenfunctions are normalized and form a complete set of functions.

\section{Semiclassical approach: generalities and equations}

\subsection{The main qualitative features of the solution}

For completeness of presentation, we start discussing the solutions to Eq. (5), repeating the key qualitative and general features of solutions that have been discussed in Ref. [1]. The first one has been mentioned in the previous section: at large values of $\kappa$, the eigenfunctions $\phi_{E}(\kappa)$ should approach the eigenfunctions of the massless BFKL equations (12).

The behavior of the solutions, at small values of $\kappa$, is easier to understand by rewriting Eq. (5) in the coordinate representation. Using

$\int \frac{\mathrm{d}^{2} p^{\prime}}{2 \pi} \frac{e^{i \mathbf{r} \cdot \mathbf{p}^{\prime}}}{p^{\prime 2}+m^{2}}=\int_{-\infty}^{+\infty} \frac{p^{\prime} \mathrm{d} p^{\prime} J_{0}\left(r p^{\prime}\right)}{p^{\prime 2}+m^{2}}=K_{0}(r m)$

where $J_{0}(z)$ and $K_{0}(z)$ are the Bessel and MacDonald functions [43], we can rewrite Eq. (5) in the form

$$
\begin{aligned}
E f(r) & =\mathscr{H} f_{E}(r), \text { where } \\
f_{E}(r) & =\int \frac{\mathrm{d}^{2} p}{(2 \pi)^{2}} e^{i \mathbf{p} \cdot \mathbf{r}} \phi_{E}\left(\frac{p^{2}}{m^{2}}\right),
\end{aligned}
$$

and

$$
\begin{aligned}
\mathscr{H}= & \frac{p^{2}+m^{2}}{|p| \sqrt{p^{2}+4 m^{2}}} \ln \frac{\sqrt{p^{2}+4 m^{2}}+|p|}{\sqrt{p^{2}+4 m^{2}}-|p|}-2 K_{0}(|r| m) \\
& +\frac{N_{c}^{2}+1}{2 N_{c}^{2}} \hat{P} \\
= & T\left(p^{2}\right)+V(r)+\frac{N_{c}^{2}+1}{2 N_{c}^{2}} \hat{P}=\mathscr{H}_{0}+\frac{N_{c}^{2}+1}{2 N_{c}^{2}} \hat{P} .
\end{aligned}
$$

In (15) we introduced a shorthand notation $\hat{P}$ for the projector onto the state $\sim m^{2} /\left(p^{2}+m^{2}\right)$,

$$
\begin{aligned}
& \hat{P} \phi(p)=\frac{m^{2}}{p^{2}+m^{2}} \int \frac{\mathrm{d}^{2} p^{\prime}}{\pi} \frac{\phi\left(p^{\prime}\right)}{p^{\prime 2}+m^{2}} \\
& \stackrel{\text { in coordinate representation }}{\longrightarrow} K_{0}(|r| m) \int \frac{\mathrm{d}^{2} p^{\prime}}{\pi} \frac{\phi\left(p^{\prime}\right)}{p^{\prime 2}+m^{2}} .
\end{aligned}
$$

The behavior of Eq. (12) at large $p$ translates into the short distance behavior

$f_{E=E(\gamma)}(r) \stackrel{r \ll 1 / m}{\longrightarrow} f_{E=E(\gamma)}^{\mathrm{BFKL}}(r) \sim\left(r^{2}\right)^{\gamma-1} \cup\left(r^{2}\right)^{-\gamma}$.

To understand the behavior of the solutions at large distances, we should distinguish the two cases, when the wave function's decrease is slower than $e^{-m r}$, and when the decreases are faster than $e^{-m r}$. In the former case, as we may see from Eqs. (15) and (16), we may neglect the contact term and the term $\sim V(r)$, and Eq. (14) degenerates into

$$
\begin{aligned}
T f_{E=E(\gamma)}^{0}(r) & =\frac{\kappa+1}{\sqrt{\kappa} \sqrt{\kappa+4}} \ln \frac{\sqrt{\kappa+4}+\sqrt{\kappa}}{\sqrt{\kappa+4}-\sqrt{\kappa}} f_{E=E(\gamma)}^{0}(r) \\
& =E(\nu) f_{E=E(\gamma)}^{0}(r)
\end{aligned}
$$

where $\kappa=-\nabla_{r}^{2}$. The eigenfunctions of Eq. (18) have the form

$f_{E=E(v)}^{0}(\mathbf{r}) \sim e^{i \sqrt{-a} r}$ for $a<0 ;$ and

$f_{E=E(v)}^{0}(\mathbf{r}) \sim e^{-\sqrt{a} r}$ for $a>0$.

From Eq. (18) we can see that the parameters $a$ and $\gamma$ are correlated, viz.

$E=T(-a)=-\chi(\gamma)$.

The solution to this equation is shown in Fig. 2. The Fourier image of $1 /\left(p^{2}+m^{2}\right)^{1-\gamma}$ in the coordinate representation is

$$
\begin{aligned}
& \frac{1}{(\kappa+a)^{1-\gamma}} \stackrel{\text { Fourier image }}{\longrightarrow} \frac{1}{\Gamma(1-\gamma)}\left(\frac{2 \sqrt{a}}{r}\right)^{\gamma} \\
& K_{\gamma}(\sqrt{a} r) \stackrel{\sqrt{a} r \gg 1}{\longrightarrow} \frac{1}{\Gamma(1-\gamma)}\left(\frac{2 \sqrt{a}}{r}\right)^{\gamma} \sqrt{\frac{\pi}{2 \sqrt{a} r}} e^{-\sqrt{a} r} .
\end{aligned}
$$

The function $1 /(\kappa+a)^{1-\gamma}$ describes both the short distance (17) and the long distance (19) behaviors. In [1] we demonstrated that the ground state with the minimal energy is achieved on this class of functions. 


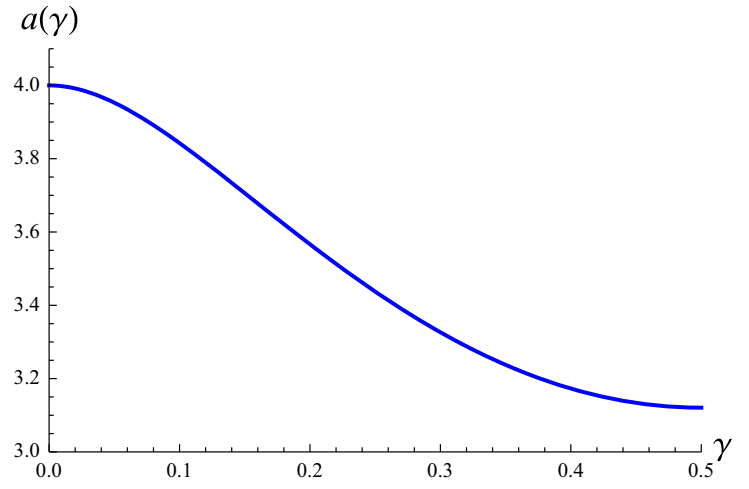

(a)

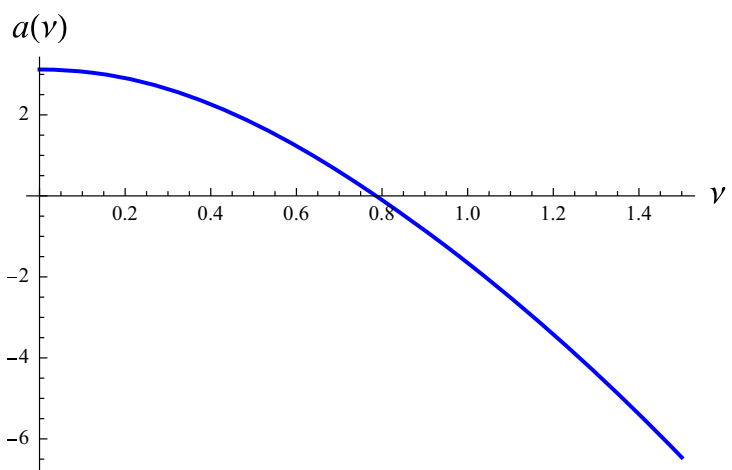

(b)

Fig. 2 The function $a(\gamma)$ (solution to Eq.(20)) versus $\gamma$ (a) and versus $v$ where $\gamma=\frac{1}{2}+i v(\mathbf{b})$

\subsection{Semiclassical approach: generalities}

\subsubsection{The method of steepest descent}

For massless BFKL, the general solution has the form

$\Psi(Y, \kappa)=\int_{\epsilon-i \infty}^{\epsilon+i \infty} \frac{\mathrm{d} \gamma}{2 \pi i} \phi_{\text {in }}(\gamma) e^{-E(\gamma) Y+(\gamma-1) \ln \kappa}$,

where $\phi_{\text {in }}(\gamma)$ should be found from the initial conditions at $Y=0$. For the massive case, we will look for solutions of Eq. (8) of the analogous form

$\Psi(Y, l)=\int_{\epsilon-i \infty}^{\epsilon+i \infty} \frac{\mathrm{d} \gamma}{2 \pi i} \phi_{\text {in }}(\gamma) e^{-E(\gamma, l) Y+(\gamma-1) l}$,

where we introduced a new variable $l=\ln (\kappa+a)$, which is stable in the small $-\kappa$ limit, and $\phi_{\text {in }}(\gamma)$ is fixed by the initial conditions at $Y=0$. In Eq. (23) we can take the integral over $\gamma$ using the method of steepest descent, which is equivalent to a search of the semiclassical solution of Eq. (8). The equation for the saddle point takes the general form

$-\frac{\partial E\left(\gamma_{\mathrm{SP}}(Y, l), l\right)}{\partial \gamma} Y+l=0$.

If $\phi_{\text {in }}(\gamma)$ in the integrand of (23) is a smooth function, we

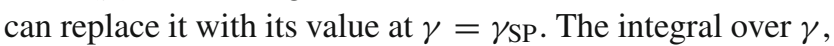
in the vicinity of a saddle point, (24), yields

$$
\begin{aligned}
& \Psi(Y, l) \\
& =\phi_{\text {in }}\left(\gamma_{\mathrm{SP}}(Y, l)\right) e^{-E\left(\gamma_{\mathrm{SP}}(Y, l), l\right) Y+\left(\gamma_{\mathrm{SP}}(Y, l)-1\right) l} \\
& \quad \times \int_{\epsilon-i \infty}^{\epsilon+i \infty} \frac{\mathrm{d} \gamma}{2 \pi i} \\
& \exp \left(-\frac{1}{2} \frac{\partial^{2} E\left(\gamma_{\mathrm{SP}}(Y, l), l\right)}{\partial \gamma_{\mathrm{SP}}^{2}} Y\left(\gamma-\gamma_{\mathrm{SP}}(Y, l)\right)^{2}\right)
\end{aligned}
$$

$$
\begin{aligned}
& =\phi_{\text {in }}\left(\gamma_{\mathrm{SP}}(Y, l)\right) \sqrt{\frac{1}{2 \pi\left|\frac{\partial^{2} E\left(\gamma_{\mathrm{SP}}(Y, l), l\right)}{\partial \gamma_{\mathrm{SP}}^{2}}\right| Y}} e^{S(Y, l)} \\
& =\phi_{\text {in }}\left(\gamma_{\mathrm{SP}}(Y, l)\right) \sqrt{\frac{1}{2 \pi\left|\frac{\partial^{2} E\left(\gamma_{\mathrm{SP}}(Y, l), l\right)}{\partial \gamma_{\mathrm{SP}}^{2}}\right| Y}} e^{-\frac{1}{2} l} e^{\omega_{\mathrm{eff}}(l, Y) Y} .
\end{aligned}
$$

The omission of higher-order corrections in Eq. (25) is justified due to the smallness of the parameter

$$
\begin{aligned}
R= & \frac{1}{6}\left|\frac{\partial^{3} E\left(\gamma_{\mathrm{SP}}(Y, l), l\right)}{\partial \gamma_{\mathrm{SP}}^{3}}\right| Y / \\
& \times\left(\frac{1}{2}\left|\frac{\partial^{2} E\left(\gamma_{\mathrm{SP}}(Y, l), l\right)}{\partial \gamma_{\mathrm{SP}}^{2}}\right| Y\right)^{3 / 2} \ll 1 .
\end{aligned}
$$

The smoothness of the initial function $\phi_{\text {in }}(\gamma)$ implies the condition

$\left.\frac{1}{\sqrt{\left|\frac{1}{2} \frac{\partial^{2} E\left(\gamma_{\mathrm{SP}}(Y, l), l\right)}{\partial \gamma_{\mathrm{SP}}^{2}}\right| Y}} \ll \frac{\ln \phi_{\text {in }}(\gamma)}{\mathrm{d} \gamma}\right|_{\gamma=\gamma_{\mathrm{SP}}}$

and determines the kinematic region of applicability of the semiclassical approximation. As we will demonstrate below, both conditions are satisfied for sufficiently large $Y \gg 1$.

\subsubsection{Solution with method of characteristics}

The method of characteristics for a partial differential equation (PDE) corresponds to a reduction of the PDEs to a system of ordinary differential equations (ODE) for characteristic lines along which the PDE converts into an ordinary differential equation. A direct application of the method of characteristics to the evolution equation, Eq. (8), is not straightforward, since it is an integrodifferential equation. However, as we show in detail in Sect.1, for the special case which corresponds to a semiclassical approximation, this method is 
applicable. The characteristic lines correspond to fixed eigenvalues $\omega$, with the rapidity $Y$ as a natural parameter, i.e.

$\omega_{\mathrm{SC}}=\chi\left(\gamma_{\mathrm{SC}}\right)+\widetilde{P}\left(l(Y), \gamma_{\mathrm{SC}}(Y), a\left(\gamma_{\infty}\right)\right)=\chi\left(\gamma_{\infty}\right)$.

where for the sake of convenience we use the new variable $l=$ $\ln (\kappa+a)$. Along each trajectory, $\gamma(l)$ satisfies a differential equation,

$$
\begin{aligned}
\frac{\mathrm{d} \gamma_{\mathrm{SC}}}{\mathrm{d} l}= & -\frac{\partial \widetilde{P}\left(l(t), \gamma_{\mathrm{SC}}(t), a\right)}{\partial l} / \\
& \left(\frac{\mathrm{d} \chi\left(\gamma_{\mathrm{SC}}\right)}{\mathrm{d} \gamma_{\mathrm{SC}}}+\frac{\partial \widetilde{P}\left(l(t), \gamma_{\mathrm{SC}}(t), a\right)}{\partial \gamma_{\mathrm{SC}}}\right),
\end{aligned}
$$

and the relation with the parameter $Y$ is given by

$$
\frac{\mathrm{d} l(Y)}{\mathrm{d} Y}=-\frac{\mathrm{d} \chi\left(\gamma_{\mathrm{SC}}(l(Y))\right)}{\mathrm{d} \gamma}-\frac{\partial \widetilde{P}\left(l(Y), \gamma_{\mathrm{SC}}(l(Y)), a\right)}{\partial \gamma_{\mathrm{SC}}} .
$$

The effective intercept introduced in Eq. (25) equals

$\omega_{\mathrm{eff}}(Y, l)=\left(S\left(Y, l_{\mathrm{SP}}(Y)\right)+\frac{1}{2} l_{\mathrm{SP}}(Y)\right) / Y$,

where $S(Y)$ and $l(Y)$ are found from

$$
\begin{aligned}
\frac{\mathrm{d} S}{\mathrm{~d} Y}= & \left(\gamma_{\mathrm{SC}}(Y, l)-1\right) \frac{\mathrm{d} l(Y)}{\mathrm{d} Y} \\
& +\chi\left(\gamma_{\mathrm{SC}}(Y, l)\right)+\widetilde{P}\left(l, \gamma_{\mathrm{SC}}(Y, l, a)\right) .
\end{aligned}
$$

Before applying the semiclassical approach to the BFKL equation for massive gluons, in Sect. 2.3, we would like to test it on the massless BFKL equation (9), for which analytical solutions are known (see for example Ref. [3,9]), and only after that in Sect. 2.4 we apply it to the massive case.

\subsection{Massless BFKL equation in semiclassical approach}

Combining Eqs. (9), (12), and (24), we obtain for the saddle point $\gamma_{\mathrm{SP}}(Y, l=\ln \kappa)$

$\chi_{\gamma}^{\prime}\left(\gamma_{\mathrm{SP}}(\xi)\right)+\xi=0$ where $\xi=l / Y$.

The solution to Eq. (33) is shown in Fig. 3a. Equation (25) yields

$$
\begin{aligned}
& \Psi_{\mathrm{BFKL}}(Y, l=\ln \kappa) \propto e^{\omega_{\mathrm{eff}}(\xi) Y}, \text { where } \\
& \omega_{\mathrm{eff}}(\xi)=\chi\left(\gamma_{\mathrm{SP}}(\xi)\right)+\left(\gamma_{\mathrm{SP}}(\xi)-\frac{1}{2}\right) \xi .
\end{aligned}
$$

The dependence $\omega_{\text {eff }}(\xi)$ is plotted in Fig. 3b. Figure 3c shows the ratio $R(\xi, Y)$ defined in Eq. (26). This ratio is small at large values of $Y$ and small $\xi$, justifying the applicability of the semiclassical approximation in this region.

\subsubsection{Diffusion approximation}

As we can see from Fig. 3a, at $\xi \rightarrow 0$ or, in other words, at large $Y \gg l$, the trajectory $\gamma_{\text {SP }}$ approaches a limiting value $\gamma_{\mathrm{SP}}(\infty)=\frac{1}{2}$. Since $\chi(\gamma)$ is an analytic function near this point, it can be approximated by

$\chi(\gamma)=\omega_{0}+D\left(\gamma-\frac{1}{2}\right)^{2}+\mathscr{O}\left(\left(\gamma-\frac{1}{2}\right)^{3}\right)$

with $\omega_{0}=4 \ln 2=2.772$ and $D=14 \zeta(3)=16.822$. In the approximation (35), Eq. (33) can be solved easily and yields

$\gamma_{\mathrm{SP}}=\frac{1}{2}-\frac{\xi}{2 D}$

Substituting (36) into Eq. (25), we obtain

$\Psi \operatorname{BFKL}(\xi, Y)=\phi_{\text {in }}\left(\frac{1}{2}-\frac{\xi}{2 D}\right) \sqrt{\frac{1}{4 \pi D Y}} e^{-\frac{1}{2} l} e^{\omega_{0} Y-\frac{\xi^{2}}{4 D Y}}$,

i.e., the semiclassical approach reproduces the diffusion approximation [9] for the BFKL equation.

\subsubsection{Double log approximation}

For small values of $\gamma \approx 0$, the BFKL kernel can be approximated by

$\chi(\gamma)=\frac{1}{\gamma}$

In this limit, Eq. (33) gives the trajectory $\gamma_{\mathrm{SP}}=1 / \sqrt{\xi}$ and the wave function

$$
\begin{aligned}
\Psi_{\mathrm{BFKL}}(\xi, Y) & =\phi_{\text {in }}(1 / \xi) \sqrt{\frac{1}{2 \pi \xi^{3 / 2} Y} e^{2 \sqrt{\xi} Y}} \\
& =\phi_{\text {in }}(Y / l) \sqrt{\frac{1}{2 \pi l^{3 / 2} Y^{-1 / 2}}} e^{2 \sqrt{Y l}} .
\end{aligned}
$$

Equation (39) is the solution in the double log approximation [9].

2.4 Massive BFKL equation in semiclassical approach

Plugging the solution Eq. (23) into Eq. (8), we obtain the equation for $E(\gamma, l)$ in the form

$E(\gamma, l)=T\left(e^{l}-a\right)+C T(l, \gamma(l), a ;)-\mathscr{K}(l, \gamma(l), a)$, 


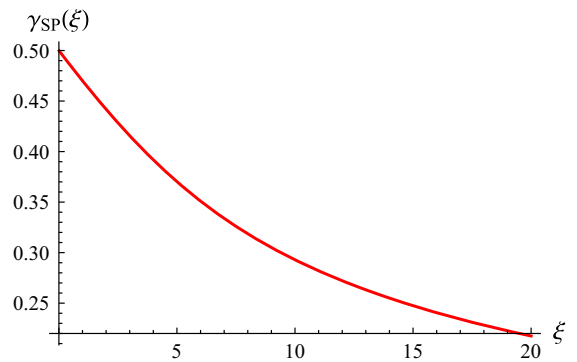

(a)

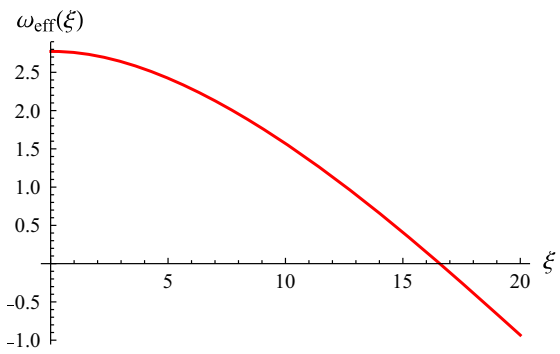

(b)

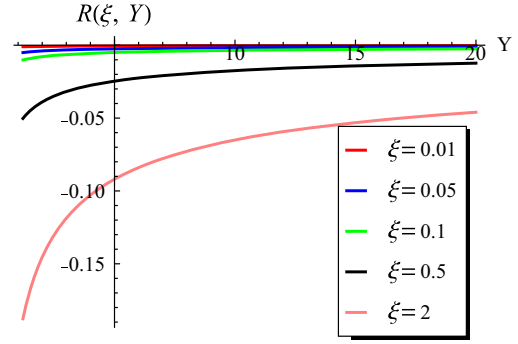

(c)

Fig. 3 Semiclassical solution for massless BFKL equation (see Eq. (9)): trajectories as a function of $\xi=l / Y$ (a); effective Pomeron intercept $\left(\omega_{\text {eff }}\right.$ versus $\xi(\mathbf{b})$; and the ratio $R$ of Eq. (26) at fixed $\xi$ as a function of $Y(\mathbf{c})$

where the emission kernel $\mathscr{K}(l, \gamma(l), a)$ is given by

$$
\begin{aligned}
\mathscr{K}(l, \gamma(l), a)= & \int_{\frac{a}{\kappa}}^{1} \frac{t^{\gamma(l)-1} \mathrm{~d} t}{\sqrt{(1-t)^{2}+\frac{2}{\kappa}(1+t)+\frac{1-4 a}{\kappa^{2}}}} \\
& +\int_{0}^{1} \frac{t^{-\gamma(l)} \mathrm{d} t}{\sqrt{(1-t)^{2}+\frac{2}{\kappa} t(1+t)+(1-4 a) \frac{t^{2}}{\kappa^{2}}}} .
\end{aligned}
$$

The integral over $t$ in Eq. (41) can be evaluated analytically and expressed in terms of the Appel function $F_{1}{ }^{1}$ :

$$
\begin{aligned}
\mathscr{K}(l, \gamma(l), a)= & 2 \sqrt{\frac{t^{0}-t^{+}}{t^{+}-t^{-}}}\left(t^{+}\right)^{1-\gamma(l)} \\
& \times F_{1}\left(\frac{1}{2}, 1-\gamma(l), \frac{1}{2}, \frac{1}{2}, 1-\frac{t^{0}}{t^{+}}, \frac{t^{+}-t^{0}}{t^{+}-t^{-}}\right) \\
& +\sqrt{\pi} \frac{\Gamma(\gamma(l))}{\Gamma\left(\frac{1}{2}+\gamma(l)\right)} 2 \\
& \times F_{1}\left(\frac{1}{2}, \frac{1}{2}, \gamma(l)-\frac{1}{2}, \frac{t^{+}}{t^{+}-t^{-}}\right) \\
& +B(1, \gamma(l)) \\
& \times F_{1}\left(\gamma(l), \frac{1}{2}, \frac{1}{2}, 1+\gamma(l), t^{-}, t^{+}\right)
\end{aligned}
$$

where

$$
t^{0}=\frac{a}{\kappa} ; \quad t^{ \pm}=1+\frac{1}{\kappa} \pm 2 \sqrt{\frac{t^{0}-1}{\kappa}} .
$$

For practical reasons it is convenient to introduce a function $P(l, \gamma, a)$ defined as

$$
\begin{aligned}
& P(l, \gamma, a)=\int_{t^{0}}^{1} \mathrm{~d} t\left(t^{\gamma-1}-1\right) \\
& {\left[\frac{1}{\sqrt{(1-t)^{2}+(2 / \kappa)(1+t)+(1-4 a) / \kappa^{2}}}\right.} \\
& \left.\quad-\frac{1}{\sqrt{(1-t)^{2}}}\right]-\int_{0}^{t^{0}} \mathrm{~d} t \frac{t^{\gamma-1}-1}{\sqrt{(1-t)^{2}}}+\int_{0}^{1} \mathrm{~d} t\left(t^{-\gamma}-1\right)
\end{aligned}
$$

$\overline{1}$ See Eqs. 9.180-9.184 in Ref. [43].

$$
\begin{aligned}
& {\left[\frac{1}{\sqrt{(1-t)^{2}+(2 / \kappa) t(1+t)+(1-4 a) t^{2} / \kappa^{2}}}\right.} \\
& \left.-\frac{1}{\sqrt{(1-t)^{2}}}\right] .
\end{aligned}
$$

Then Eq. (40) can be cast into the form

$$
\begin{aligned}
E= & -\chi(\gamma(l))+\tilde{T}(l, a)+C T(l, \gamma(l), a) \\
& -P(l, \gamma(l), a)=-\chi(\gamma(l))-\widetilde{P}(l, \gamma(l), a),
\end{aligned}
$$

where

$$
\begin{aligned}
& \mathrm{CT}(l, \gamma, a)=\frac{5}{9} \frac{e^{(1-\gamma) l}}{e^{l}+1-a}(a-1)^{1+\gamma} B\left(\frac{a-1}{a}, 1-\gamma, a\right), \\
& \tilde{T}(l, a)=T\left(e^{l}-a\right)-L\left(e^{l}, a\right) \\
& L(\kappa, a)=\int_{\frac{a}{\kappa}}^{1} \frac{\mathrm{d} t}{\sqrt{(1-t)^{2}+\frac{2}{\kappa}(1+t)+\frac{1-4 a}{\kappa^{2}}}} \\
& +\int_{0}^{1} \frac{\mathrm{d} t}{\sqrt{(1-t)^{2}+\frac{2}{\kappa} t(1+t)+(1-4 a) \frac{t^{2}}{\kappa^{2}}}}, \\
& =-\ln \left(1+a-\kappa+\sqrt{(1-a+\kappa)^{2}}\right) \\
& +\ln (1+\sqrt{1-4 a+4 \kappa)} \\
& -\frac{\kappa \ln \left(\kappa\left(1-\kappa+\sqrt{-4 a+(1+\kappa)^{2}}\right)\right.}{\sqrt{-4 a+(1+\kappa)^{2}}} \\
& +\frac{\kappa \ln \left(1-4 a+3 \kappa+\sqrt{1-4 a+4 \kappa} \sqrt{-4 a+(1+\kappa)^{2}}\right)}{\sqrt{-4 a+(1+\kappa)^{2}}}
\end{aligned}
$$

and $B(x, p, q)$ is the incomplete Beta function. ${ }^{2}$ Figure 4 illustrates how all the ingredients of Eq. (45) behave as functions of $l=\ln (\kappa+a(v))$.

\footnotetext{
$\overline{2 \text { See Eq. } 8.39}$ in Ref. [43].
} 


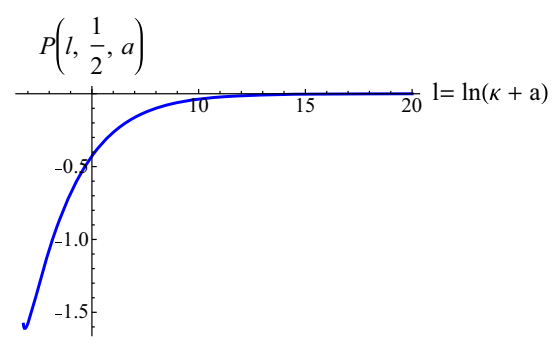

(a)

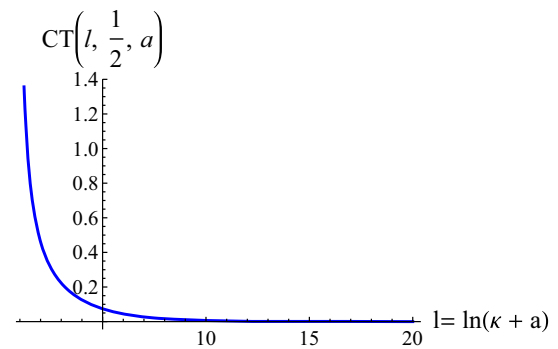

(b)

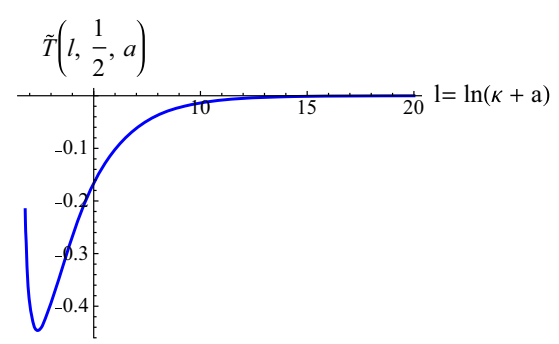

(c)

Fig. 4 All ingredients of Eq. (4) versus $l$ at $\gamma=\frac{1}{2}$ and $a=a\left(\frac{1}{2}\right)$ (see Eq. (20); Fig. 2)

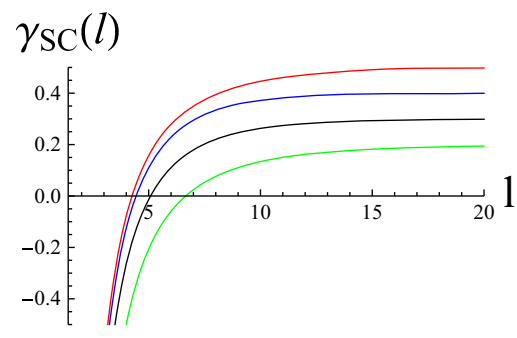

(a)

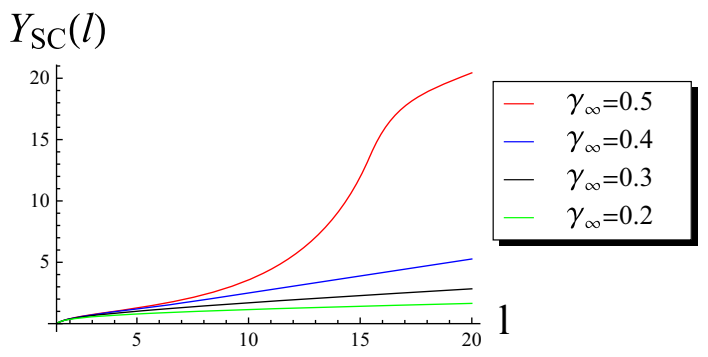

(b)

Fig. 5 The trajectories for the BFKL equation with massive gluons (solutions to Eq. (28) and to Eq. (30)) versus $l=\ln (\kappa+a)$. The function $Y_{\mathrm{SC}}(l)$ is the inverse function to $l=l(Y)$ of Eq. (29)

\section{Semiclassical solutions to the BFKL equation with massive gluon: numerical results}

\subsection{Trajectories and intercepts}

To solve the general BFKL equation with massive gluons, we need to find the trajectory from Eq. (24) which will be a function of both $\xi=l / Y$ and $l$. According to the method of characteristics discussed in Sect. 2.2.2, these trajectories are the solutions of Eq. (28) or, equivalently, Eq. (29). Unfortunately, we are able to solve Eq. (28) only numerically, and these solutions are shown in Fig. 5.

All the trajectories can be characterized by their asymptotic boundary condition $\gamma_{\mathrm{SC}}(l) \stackrel{l \gg 1}{\longrightarrow} \gamma_{\infty}$. We note that in Eq. (20) one should understand $\gamma$ as $\gamma_{\infty}$, thus reducing it to the form

$T(-a)=-\chi\left(\gamma_{\infty}\right)$.

At small values of $l$ all the trajectories $\gamma_{\mathrm{SC}}(l, \xi)$ vanish, reflecting the $K_{0}(\sqrt{a} r)$-behavior of the solution in the coordinate representation. The negative value of $\gamma_{\mathrm{SC}}$ for soft $l$ in a numerical solution of Eq. (28) is different from what one expects from a massless BFKL. In Sect. 3.4.3 we will discuss this region in more detail, here we only point out that $E(\gamma, l)$ is an analytical function of $\gamma$ for negative values of $\gamma$ 's without any singularities at $\gamma=-n, n=0,1,2 \ldots$. Figure 6 shows that $\gamma_{\mathrm{SP}}(l)$, given by the solution of Eq. (28) and presented in Fig. 5, satisfies the equation even at $l<l_{\text {soft }}$.

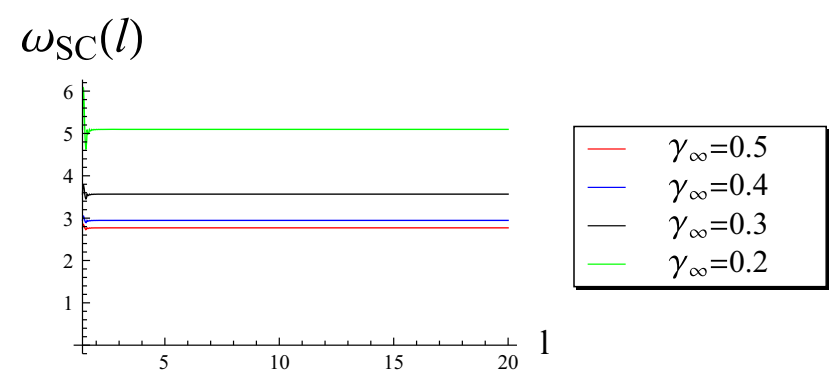

Fig. 6 Intercept of the massive BFKL Pomeron versus $l$ on the trajectories with different $\gamma_{\infty}$

The trajectories $l_{\mathrm{SP}}(Y)$ can be found by solving Eq. (29), and they are shown in Fig. 5b for several boundary conditions. In the $\xi \ll 1$ region the solution may be constructed analytically without solving an additional equation. The first observation is that at large $l$, the set of trajectories should coincide with the same set for the massless BFKL equations (33),

$\gamma_{\mathrm{SP}}(l, \xi) \stackrel{l \gg 1}{\longrightarrow} \gamma_{\mathrm{SP}}^{\mathrm{BFKL}}(\xi) \equiv \gamma_{\infty}$.

Assuming that $\gamma_{\mathrm{SP}}^{\mathrm{BFKL}}(\xi)$ is close to $\frac{1}{2}$ at small $\xi$ (the socalled Bjorken limit), we can consider the deviation as a small parameter,

$\gamma_{\mathrm{SP}}(l)=\gamma_{\mathrm{SC}}\left(l, \gamma_{\infty}=\frac{1}{2}\right)+\delta \gamma_{\mathrm{SP}}(l, \xi)$.

To find $\delta \gamma_{\mathrm{SP}}(l, \xi)$, Eq. (24) can be rewritten in the form 


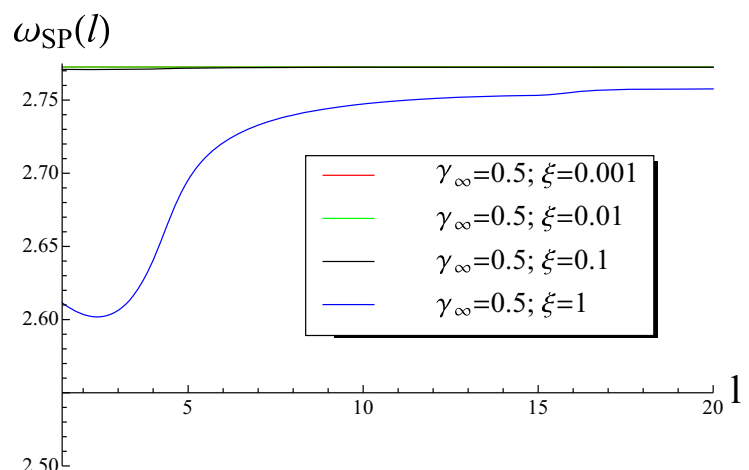

(a)

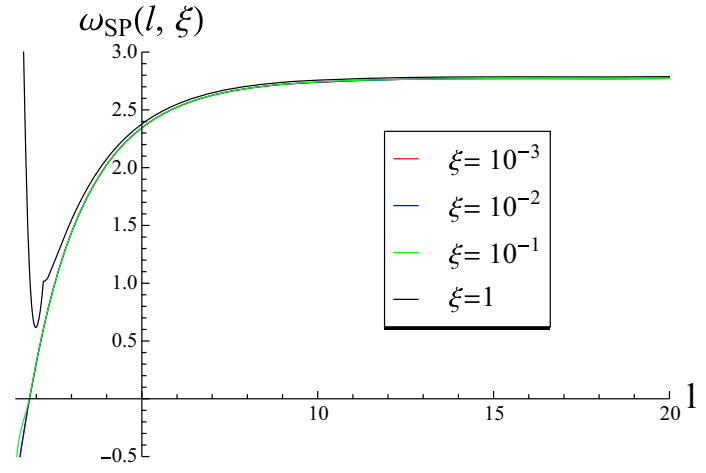

(b)

Fig. $7 \omega_{\mathrm{eff}} \equiv \omega_{\mathrm{SP}}$ of the massive BFKL Pomeron versus $l$ at fixed $\xi=l / Y$. b $\omega_{\mathrm{SP}}(l)$ are close for small values of $\xi$

$$
\begin{aligned}
& \delta \gamma_{\mathrm{SP}}(l)=\lim _{\epsilon \rightarrow 0} \\
& \left(-\xi \frac{\left(\gamma_{\mathrm{SC}}\left(l, \gamma_{\infty}=\frac{1}{2}\right)+\epsilon-\frac{1}{2}\right)}{\left(\frac{\mathrm{d} \chi\left(\gamma=\gamma_{\mathrm{SC}}\left(l, \gamma_{\infty}=\frac{1}{2}\right)+\epsilon\right)}{\mathrm{d} \gamma}+\frac{\partial \widetilde{P}\left(l, \gamma=\gamma_{\mathrm{SC}}\left(l, \gamma_{\infty}=\frac{1}{2}\right)+\epsilon, a\left(\gamma_{\infty}\right)\right)}{\partial \gamma}\right)}\right),
\end{aligned}
$$

where $\epsilon \approx 0$ is a small cutoff needed to regularize some intermediate results. In particular, Eq. (53) reproduces Eq. (36) and gives the final result at $l \gg 1$ where

$$
\frac{\mathrm{d} \chi\left(\gamma=\gamma_{\mathrm{SP}}(l)\right)}{\mathrm{d} \gamma}+\frac{\partial \widetilde{P}\left(l, \gamma=\gamma_{\mathrm{SC}}(l), a\left(\gamma_{\infty}\right)\right)}{\partial \gamma} \ll 1 .
$$

The solution to Eq. (24) in this approximation has the form

$$
\begin{aligned}
& \Psi(Y, l) \\
& =\phi_{\text {in }}\left(\gamma_{\mathrm{SP}}(Y, l)\right) \sqrt{\frac{1}{2 \pi\left|\frac{\partial^{2} E\left(\gamma_{\mathrm{SP}}(Y, l), l\right)}{\partial \gamma_{\mathrm{SP}}^{2}}\right| Y}} e^{-\frac{1}{2} l} e^{\omega_{\mathrm{eff}}(l, \xi) Y},
\end{aligned}
$$

where we introduced an effective intercept,

$\omega_{\mathrm{eff}}(l, \xi)=-E\left(\gamma_{\mathrm{SP}}(Y, l), l\right)+\left(\gamma_{\mathrm{SP}}(Y, l)-\frac{1}{2}\right) \xi$,

and $\gamma_{\text {SP }}$ is given by Eq. (52).

The $l$-dependence of the effective intercepts $\omega(l, \xi)$ is shown in Fig. 7. At small $\xi$ these intercepts are close to the massless BFKL given by Fig. 3a in the entire region of $l$. In the region of large $\xi$, the effective intercepts are considerably smaller than the intercept of the massless BFKL Pomeron. For small $l$, i.e., the scattering amplitude at small values of $l$ and large values of $\xi$, it is suppressed in comparison to the behavior determined by the intercept of the massless BFKL Pomeron. Since the asymptotic region at high energies (large $Y$ ) corresponds to $\xi \ll 1$, the high energy behavior of the massive BFKL Pomeron is the same as for the massless one. This agrees with our earlier results of the numerical calculation (see Ref. [1]).

\subsection{Accuracy of the semiclassical approach}

The accuracy of the semiclassical approximation is controlled by the ratio $R$ of Eq. (26). Figure 8 shows this ratio for different values of $\xi$. From the figure we conclude that for $\xi \leq 1$ the ratio $R$ is small and we can safely use the semiclassical approximation. For $l<4$, the ratio $R$ is small in the region of large $Y$ (small $\xi$ ). This occurs because for large $l$ the solution of the BFKL equation for the massive gluons should coincide with the solution of the massless BFKL equation.

These estimates confirm our expectation that the semiclassical method provides a reliable approach at high energies (large values of $Y$ ).

At large $\xi$, our procedure does not work even at large $l$. In terms of kinematics, we need to deal with $\gamma_{\text {SC }} \ll 1$, while in Fig. 8 we used $\gamma_{\mathrm{SC}} \stackrel{l \gg 1}{\longrightarrow} \frac{1}{2}$. For this region of large $l$ and $\xi$, we develop an approximation which corresponds to the double log approximation and for very large $l$ it coincides with the DLA for the massless BFKL equation.

In Fig. 9 we plot the ratio

$$
R_{\mathrm{SC}}=\frac{\frac{\mathrm{d} \gamma_{\mathrm{SC}}(l)}{\mathrm{d} l}}{\left(1-\gamma_{\mathrm{SC}}(l)\right)^{2}}
$$

This parameter controls the smoothness of the functions $\omega(Y, l)$ and $\gamma(Y, l)$ and thus the precision of the semiclassical approach. Figure 9 shows that this ratio is very small at large $l$ and does not exceed $\approx 0.4$ even at small $l$.

\subsection{Saturation momentum}

It is well known that we can find a saturation momentum by searching for the particular trajectory on which the wave 


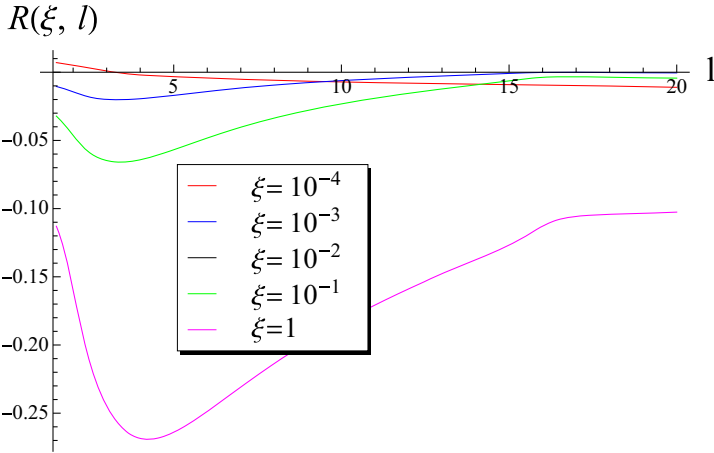

(a)

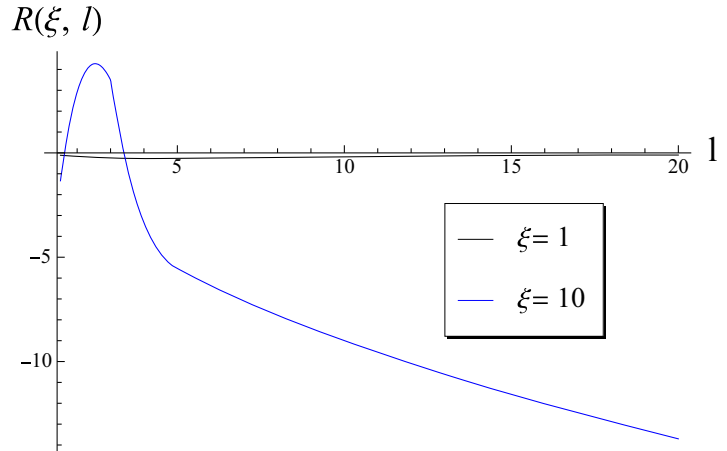

(b)

Fig. 8 The ratio of Eq. (26) for large values of $Y$. a Ratio at fixed $\xi=l / Y$ which are small, and $\mathbf{b} R$ at $\xi=1$ as a function of $l$

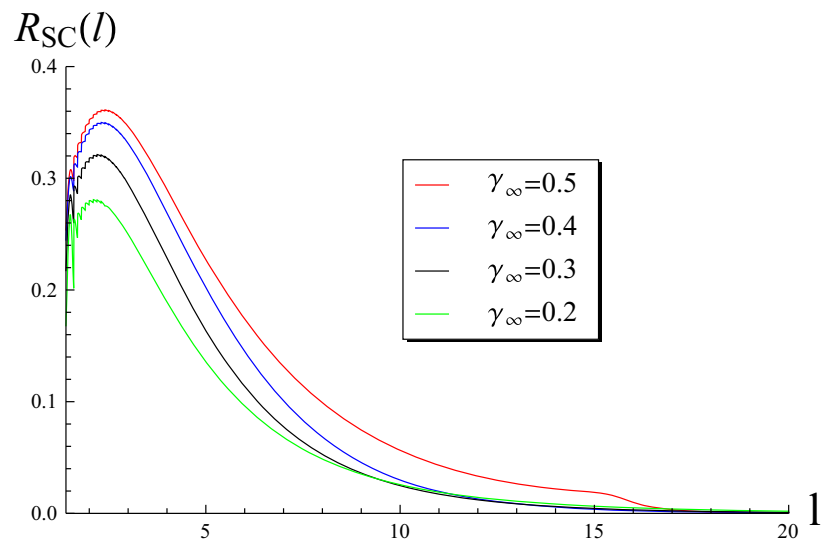

Fig. 9 The ratio of Eq. (57) versus $l$

function $\Psi(Y, \kappa)=$ const (see Refs. [3,44-48]). Mathematically, this corresponds to a solution of the system of two equations

trajectory: $-\frac{\partial E\left(\gamma_{\mathrm{SP}}(Y, l), l\right)}{\partial \gamma} Y+l=0$,

front line: $E\left(\gamma_{\mathrm{SP}}(Y, l), l\right) Y=\left(1-\gamma_{\mathrm{SP}}\right) l$.

In the case of the massless BFKL equation, the solution to the equations of Eq. (58) is $\gamma_{\mathrm{SP}}=\gamma_{\mathrm{cr}}=0.37$ [3]. For the massive BFKL equation, the critical trajectory is shown in Fig. 10a. The equation for the saturation momentum for massless BFKL equations is of the form

$l_{\mathrm{cr}}=\ln \left(Q_{s}^{2}(Y) / Q_{s}^{2}(Y=0)\right)=\frac{\chi\left(\gamma_{\mathrm{cr}}\right)}{1-\gamma_{\mathrm{cr}}} Y$.

The solution to Eq. (58) for $l_{\mathrm{cr}}=\ln \left(Q_{s}^{2}(Y) / Q_{s}^{2}(Y=0)\right)$ is shown in Fig. 10b. The difference between the massive and massless cases is sizable only for small values of $l=$ $\ln (\kappa+a)$.

\subsection{Analytical solutions}

In this section we develop two analytical methods of searching for solutions based on the diffusion and DLA approximations for the massless BFKL equation.

\subsubsection{Diffusion approximation}

A brief glance at the trajectories for small values of $\xi$ (see Fig. 6) allows us to conclude that these trajectories are close to $\gamma=\frac{1}{2}$ at least for $l \geq 5$. Therefore, for such values of $l$ we can develop the diffusion approach, in complete analogy with the case of the massless BFKL equation that has been discussed in Sect. 2.3.1. In the vicinity of $\gamma=\frac{1}{2}$ we can expand the general expression of Eq. (45) as

$$
\begin{aligned}
\omega(\gamma, l)= & -E(\gamma, l, a)=\Delta(l)+\Delta_{1}(l)\left(\gamma-\frac{1}{2}\right) \\
& +\Delta_{2}(l)\left(\gamma-\frac{1}{2}\right)^{2} .
\end{aligned}
$$

The functions $\Delta(l), \Delta_{1}(l)$ and $\Delta_{2}(l)$ are plotted in Fig. 11a.

We see that at large $l$ (say at $l \geq l_{0} \approx 5$ ) the functions $\Delta_{i}$ reach constant values, $\Delta(l) \stackrel{l>l_{0}}{\longrightarrow} \omega_{0} ; \Delta_{1}(l) \stackrel{l>l_{0}}{\longrightarrow} 0$; and $\Delta_{2}(l) \stackrel{l>l_{0}}{\longrightarrow} D$. Substituting the expansion (60) into Eq. (28), we obtain

$$
\begin{aligned}
& \Delta(l)+\Delta_{1}(l)\left(\gamma_{\mathrm{SC}}(l)-\frac{1}{2}\right) \\
& +\Delta_{2}(l)\left(\gamma_{\mathrm{SP}}-\frac{1}{2}\right)^{2}=\chi\left(\frac{1}{2}\right),
\end{aligned}
$$

whose solution is

$$
\begin{aligned}
\gamma_{\mathrm{SC}}(l) \equiv \gamma_{D}(l) & \\
= & \left(-\Delta_{1}(l) \pm \sqrt{\Delta_{1}^{2}(l)-4 \Delta_{2}(l)\left(\Delta(l)-\chi\left(\frac{1}{2}\right)\right)}\right) / \\
& \left(2 \Delta_{2}(l)\right) .
\end{aligned}
$$




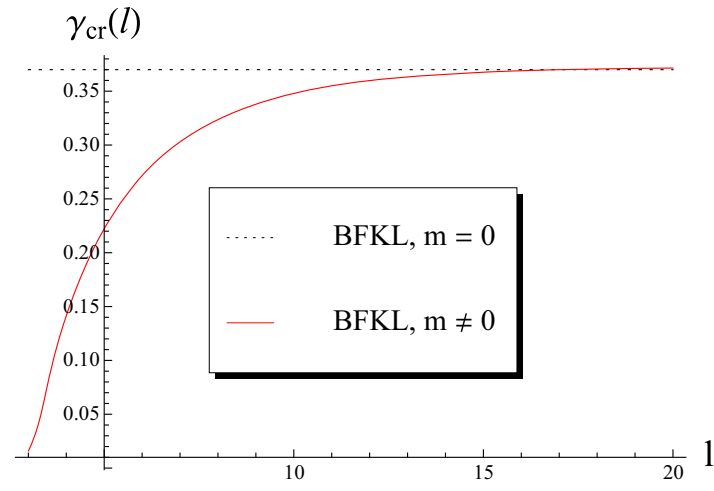

(a)

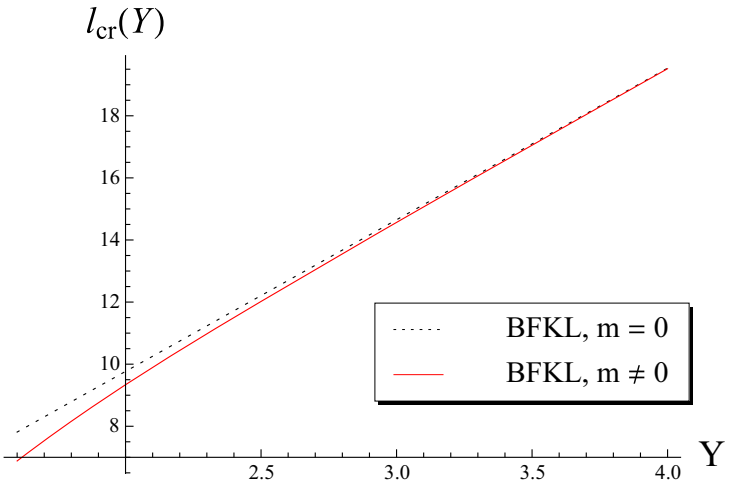

(b)

Fig. 10 The critical trajectories for BFKL equation with $m=0$ and with $m \neq 0$ (see Fig. 10a). Figure 10b shows the evolution of the logarithm of the saturation scale as defined in (59) with rapidity $Y$

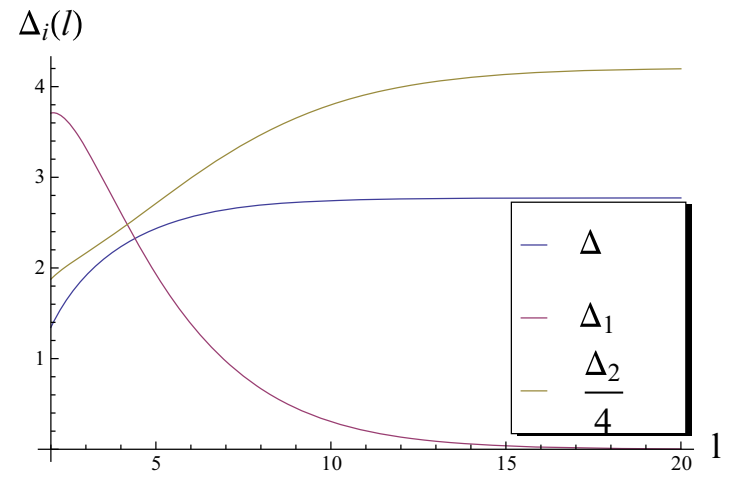

(a)

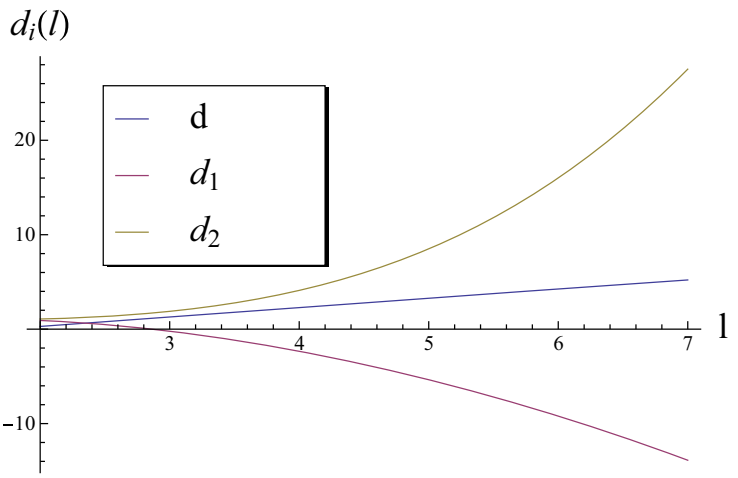

(b)

Fig. 11 Functions $\Delta_{i}(l)$ of Eq. (60) (a) and functions $d_{i}(l)$ in Eq. (69) (b)

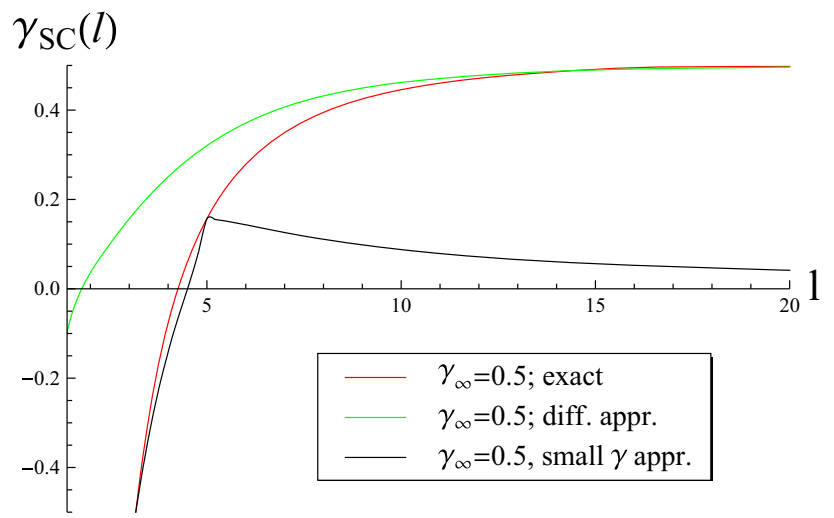

Fig. 12 Functions $\gamma_{\mathrm{SC}}(l, \xi)$ versus $l$ : exact solution to Eq. (28); diffusion approximation and approximation at small values of $\gamma$. In the figure $\xi=0.01$

The physical $\gamma_{D}$ corresponds to the minus sign in Eq. (62). In Fig. 12 we compare the trajectory Eq. (62) with the exact trajectory at $\xi=0.01$, which has been calculated in Sect. 3.1. For $l>l_{0} \sim 10$ we can safely use the solution Eq. (62).

Using Eq. (61), we can calculate the wave function of Eq. (25), which takes the form
$\Psi(Y, l)=\phi_{\text {in }}\left(\gamma_{\mathrm{SP}}(l)\right) \sqrt{\frac{1}{4 \Delta_{2}(l) Y}} e^{-\frac{1}{2} l} e^{\omega_{\mathrm{eff}}(l, \xi) Y}$,

with

$\omega_{\text {eff }}(l, \xi)=\chi\left(\frac{1}{2}\right)-\frac{\left(\xi\left(\gamma_{D}(l)-\frac{1}{2}\right)\right)^{2}}{2\left(\Delta_{1}(l)+2 \Delta_{2}(l)\left(\gamma_{D}(l)-\frac{1}{2}\right)\right)}$.

Equation (64) provides a good description of the intercept for rather large $l>l_{0} \sim 10$.

\subsubsection{Small $\gamma$ approximation}

For $l<l_{0}$, we cannot use the diffusion approximation since, as we can see from Fig. 12, the diffusion trajectory is much larger than the exact one in this region. Actually, the exact $\gamma_{\text {SP }}(l, \xi)$ at small $\xi$ approaches $\gamma_{\text {SP }} \rightarrow 0$. At $\gamma \rightarrow 0$ the general expression for $P(l, \gamma, a)$ in Eq. (45) can be simplified and takes the form

$\chi(\gamma)+\tilde{P}(l, \gamma, a) \stackrel{\gamma \rightarrow 0}{\longrightarrow} \frac{1}{\gamma}$ 


$$
+\tilde{P}_{\gamma \rightarrow 0}(l, \gamma, a)=\frac{1}{H(l, a)} \frac{1}{\gamma}\left(1-e^{-\gamma\left(l-l_{a}\right)}\right),
$$

with $H(l, a)=1 / \sqrt{(1+\exp (-l))^{2}-4 a \exp (-2 l)}$

$$
\text { and } l_{a}=\ln a \text {. }
$$

Equation (24) takes the form

$$
\begin{aligned}
& \frac{1}{H(l, a)}\left\{-\frac{1}{\gamma_{\mathrm{SC}}^{2}}\left(1-e^{-\gamma_{\mathrm{SC}}\left(l-l_{a}\right)}\right)\right. \\
& \left.+\left(l-l_{a}\right) \frac{1}{\gamma_{\mathrm{SC}}} e^{-\gamma_{\mathrm{SC}}\left(l-l_{a}\right)}\right\}=-\xi .
\end{aligned}
$$

Equation (66) has two solutions in different regions: (1) $\gamma \ll 1$ but $\gamma\left(l-l_{a}\right) \gg 1$; and (2) $\gamma \ll 1$ and $\gamma\left(l-l_{a}\right) \ll$ 1. In the first kinematic region Eq. (66) reduces to

$$
-\frac{1}{\gamma_{\mathrm{SC}}^{2}}=H(l, a)
$$

with the solution

$$
\gamma_{\mathrm{SC}}=\sqrt{H(l, a) \xi}
$$

We can check that $\gamma\left(l-l_{a}\right)=\frac{1}{\sqrt{H(l, a) \xi}}\left(l-l_{a}\right) \stackrel{l \gg l_{a}}{\longrightarrow}$ $\sqrt{\frac{Y l}{H(l, a)}} \gg 1$. Therefore, this solution corresponds to the DLA approximation in this kinematic region.

For the kinematic region $\gamma \ll 1$ and $\gamma\left(l-l_{a}\right) \ll 1$ Eq. (65) leads to the analytical function at $\gamma \rightarrow 0$, in contrast to the case of the massless BFKL kernel. Therefore, we can search for a parametrization $\omega(l, \xi)$, which has the same form as Eq. (61), viz.

$\omega_{\gamma \ll 1}(\gamma, l)=-E(\gamma, l, a=4)=\mathrm{d}(l)+\mathrm{d}_{1}(l) \gamma+\mathrm{d}_{2}(l) \gamma^{2}$,

with the functions $d_{i}$ plotted in Fig. 11b. We can see from Fig. 12 that we can rely on Eq. (69) only for $l-l_{a} \ll 1 /(\gamma=$ $0.2) \approx 5$. Equation (24) with $\omega(l, \xi)$ given by Eq. (69) has the solution

$$
\begin{aligned}
& \gamma_{\mathrm{SC}}(l) \equiv \gamma_{S}(l) \equiv \gamma_{D}(l) \\
& =\left(-\mathrm{d}_{1}(l) \pm \sqrt{\mathrm{d}_{1}^{2}(l)-4 \mathrm{~d}_{2}(l)\left(\mathrm{d}(l)-\chi\left(\frac{1}{2}\right)\right)}\right) / \\
& \left(2 \mathrm{~d}_{2}(l)\right),
\end{aligned}
$$

which is shown in Fig. 12. This solution leads to good approximation for $l=3 \div 5$ with

$$
\omega_{\mathrm{eff}}(l, \xi) \approx \chi\left(\frac{1}{2}\right)-\frac{\xi^{2} \gamma_{S}^{2}(l)}{2\left(\mathrm{~d}_{1}(l)+2 \mathrm{~d}_{2}(l) \gamma_{S}(l)\right)} .
$$

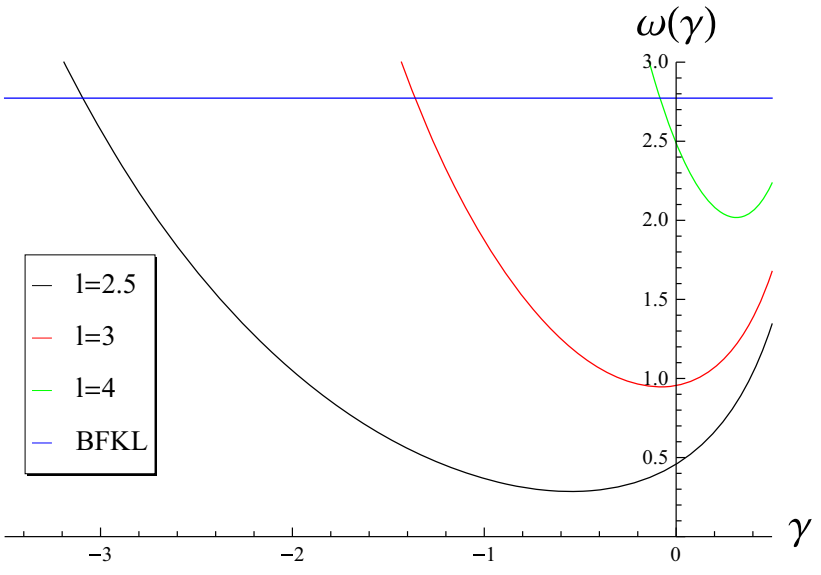

Fig. 13 Function $\omega(\gamma, l)$ versus $\gamma$ at fixed $l . \omega_{\mathrm{BFKL}}=\chi\left(\frac{1}{2}\right)$

The wave function takes the form

$\Psi(Y, l)=\phi_{\text {in }}\left(\gamma_{\mathrm{SP}}(l)\right) \sqrt{\frac{1}{4 \mathrm{~d}_{2}(l) Y}} e^{-l} e^{\omega_{\mathrm{eff}}(l) Y}$.

\subsection{3 $l \leq l_{\text {soft }}$}

Earlier we have seen that the trajectory $\gamma_{\mathrm{SP}}(l)$ has a node at some value $l_{\text {soft }}$, and becomes even negative for $l \lesssim l_{\text {sof } t}$. As we can see from (69) and Fig. 13, the function $\omega_{\mathrm{SP}}(\gamma)$ is analytic at $\gamma=0$, so we can extrapolate it to negative but small $\gamma$ 's using Eq. (69). In principle, we can expect some high twists singularities at $\gamma=-n$ with $n=0,1,2, \ldots$, which stem from the expansion of

$\frac{1}{\sqrt{(1-t)^{2}+(2 / \widetilde{\kappa})(1+t)+(1-4 a) / \widetilde{\kappa}^{2}}}=\sum_{n=0}^{\infty} C_{n}(l, a) t^{n}$

at small $t$ in Eq. (44). Taking the integral over $t$ in Eq. (44) in the vicinity of small $t$, one can see that for $\gamma \rightarrow-n$ we obtain the same expression as in Eq. (65), replacing $\gamma$ in Eq. (65) by $\gamma+n$. The energy (intercept) turns out to be a regular function at $\gamma=-n$, and we can use Eq. (69) for $|\gamma+1|<1$ with the function $d_{i}^{(1)}(l)$ calculated at $\gamma=-1$ (see Fig. 12 and Fig. 13).

Figure 13 illustrates that the intercept $\omega(\gamma, l)$ is an analytical function without singularities at negative $\gamma$ which increases at large $|\gamma|$.

Unfortunately, we have not found a simple analytical approach that enables us to describe the scattering amplitude at all values of $l$. However, we would like to recall that the numerical solutions to Eq. (52) and Eq. (53) depend neither on the value of the QCD coupling, nor on the initial condition, and reduce the procedure of calculation of the scattering amplitude to a simple equation. Solving this equation is a 
much easier task than the exact numerical calculation of the eigenvalues and eigenfunctions that was done in Ref. [1].

\section{Conclusions}

In our previous paper (see Ref. [1]) we studied the BFKL equation with massive gluons in the lattice and proved that its spectrum coincides with the spectrum of the massless BFKL equation. This observation gives rise to the hope that the correct large impact parameter $(b)$ behavior of the scattering amplitude $A \propto \exp (-m b)$, which is the inherent feature of the massive BFKL equation, will not affect the high energy behavior of the scattering amplitude. Therefore, we may expect that the modification of the BFKL equation due to confinement would not strongly affect the equations that govern the physics at high energy (in particular, the nonlinear equations of the $\mathrm{CGC} /$ saturation approach to high density QCD).

In this paper we developed the semiclassical approximation which allowed us to investigate the high energy behavior of the scattering amplitude. The method provides a simple procedure for the calculation and reduces it to a numerical solution of Eq. (28), which is much simpler than the direct numerical calculations of the eigenvalue problem in the lattice realized in Ref. [1].

Having these solutions, we propose a modification of the high energy asymptotic behavior, caused by the correct large $b$ exponential fall off of the amplitude. Actually, we did not find any unexpected behavior, and the semiclassical solution reproduces the scattering amplitude which is very close to the amplitude of the massless BFKL equation, at least at high energies.

In Sect. 3.3 we estimate the value of the saturation momentum, solving the linear evolution equation with very general assumptions as regards the nonlinear corrections. We demonstrated that the value of the saturation momentum is close to the one for the massless BFKL equations, leading to the assumption that saturation physics will look similar for both massive and massless BFKL equations.

We believe that in this paper we have taken the natural next step in the understanding of the influence of the correct large $b$ decrease of the amplitude on its high energy behavior. It should be stressed that this behavior is interesting as it provides the high energy amplitude for the electroweak-weak theory, which can be measured experimentally. The solution which has been discussed in this paper determines the asymptotic high energy behavior of the electroweak-weak theory for zero Weinberg angle. We plan to address the physical case of nonzero Weinberg angle [2] elsewhere.

Acknowledgments We thank our colleagues at UTFSM and Tel Aviv university for encouraging discussions. This research was supported by the BSF Grant 2012124 and by the Fondecyt (Chile) Grants 1140842 and 1140377.Powered@NLHPC: This research was partially supported by the supercomputing infrastructure of the NLHPC (ECM-02).

Open Access This article is distributed under the terms of the Creative Commons Attribution 4.0 International License (http://creativecomm ons.org/licenses/by/4.0/), which permits unrestricted use, distribution, and reproduction in any medium, provided you give appropriate credit to the original author(s) and the source, provide a link to the Creative Commons license, and indicate if changes were made. Funded by $\mathrm{SCOAP}^{3}$.

\section{Appendix A: Solution with method of characteristics}

The method of characteristics for a partial differential equation (PDE) corresponds to a reduction of the PDEs

$F\left(x_{1}, \ldots, x_{n}, u, p_{1}, \ldots, p_{n}\right)=0, \quad p_{i}=\frac{\partial u}{\partial x_{i}}$

to a system of ordinary differential equations (ODEs) for characteristic lines along which the PDE converts into an ordinary differential equation. These characteristics satisfy the Lagrange-Charpit equations

$\frac{\dot{x}_{i}}{F_{p_{i}}}=-\frac{\dot{p}_{i}}{F_{x_{i}}+F_{u} p_{i}}=\frac{\dot{u}}{\sum p_{i} F_{p_{i}}}$.

An instructive example, familiar from classical mechanics, is the Hamilton-Jacobi equation, for which the characteristic lines correspond to the trajectories of particles which are solutions of the Newtonian equations of motion. A detailed discussion of the method is beyond the scope of the present paper and can be found in the literature (see e.g. textbooks [49,50]).

A direct application of the method of characteristics to the evolution equation (8) is not straightforward, since it is integrodifferential equation. However, as we will show below, for a special case which corresponds to a semiclassical approximation, this method is applicable. It is convenient to rewrite the wave function $\Psi$ in terms of the "action" $S$ [51],

$\Psi(Y, \kappa)=e^{S(Y, \kappa)}$.

Then the evolution equation (8) takes the form of a nonlinear PDE,

$$
\begin{aligned}
F(Y, l, S, \gamma, \omega)= & \omega(Y, l)-\chi(\gamma(Y, l)) \\
& -\widetilde{P}(l, \gamma(Y, l, a))=0,
\end{aligned}
$$

where we introduced the shorthand notations

$\omega(Y, l)=\frac{\partial S(Y ; l)}{\partial Y} ; \quad \gamma(Y, l)-1=\frac{\partial S(Y ; l)}{\partial l}$ 
and introduced a new variable $l=\ln (\kappa+a)$ which remains finite in the small- $\kappa$ limit. The explicit form of the function $\widetilde{P}(l, \gamma(Y, l, a))$ in Eq. (A.5) is given in Sect. 2.4, and in the special limit $\widetilde{P}(l, \gamma(Y, l, a)) \stackrel{l \gg 1}{\longrightarrow} 0$. From the exact solutions of the massless BFKL (12), we expect that, at large $\kappa$, the effective action should depend linearly on the rapidity $Y$ and the new variable $l$,

$S_{\mathrm{SC}} \approx \omega_{\infty}+\left(\gamma_{\infty}-1\right) l$,

where $\gamma_{\infty}$ and $\omega_{\infty}=\omega_{\mathrm{BFKL}}\left(\gamma_{\infty}\right)$ are constants. In a semiclassical approximation which is valid for moderate values of $Y$, we assume that ${ }^{3} \omega_{\mathrm{SC}}$ and $\gamma_{\mathrm{SC}}$ are slowly varying functions of the variables $l, Y$ : viz. $\omega_{Y}^{\prime}(Y, l) \ll \omega^{2}(Y, l)$, $\omega_{l}^{\prime}(Y, l) \ll \omega^{2}(Y, l)$ and $\gamma_{Y}^{\prime}(Y, l) \ll(1-\gamma(Y, l))^{2}$, $\gamma_{l}^{\prime}(Y, l) \ll(1-\gamma(Y, l))^{2}$. Making this assumption, the equation (A.4) has the form of a PDE, which can be solved using the method of characteristics $[49,50]$. The characteristic lines $l(t), Y(t), S(t), \omega(t)$, and $\gamma_{\mathrm{SC}}(t)$, where $t$ is some parameter (the analog of time in the case of classical mechanics), satisfy the system of ODEs

$$
\begin{aligned}
& \frac{\mathrm{d} l}{\mathrm{~d} t}=\frac{\partial F}{\partial \gamma}=-\frac{\mathrm{d} \chi\left(\gamma_{\mathrm{SC}}\right)}{\mathrm{d} \gamma_{\mathrm{SC}}}-\frac{\partial \widetilde{P}\left(l(t), \gamma_{\mathrm{SC}}(t), a\right)}{\partial \gamma_{\mathrm{SC}}}, \\
& \frac{\mathrm{d} Y(t)}{\mathrm{d} t}=\frac{\partial F}{\partial \omega}=1, \\
& \frac{\mathrm{d} S}{\mathrm{~d} t}=(\gamma-1) \frac{\partial F}{\partial \gamma}+\omega \frac{\partial F}{\partial \omega} \\
& =\left(\gamma_{\mathrm{SC}}-1\right)\left\{-\frac{\mathrm{d} \chi\left(\gamma_{\mathrm{SC}}\right)}{\mathrm{d} \gamma}-\frac{\partial \widetilde{P}\left(l(t), \gamma_{\mathrm{SC}}(t), a\right)}{\partial \gamma}\right\}+\omega_{\mathrm{SC}},
\end{aligned}
$$

$\frac{\mathrm{d} \gamma_{\mathrm{SC}}}{\mathrm{d} t}=-\left(\frac{\partial F}{\partial l}+\left(\gamma_{\mathrm{SC}}-1\right) \frac{\partial F}{\partial S}\right)$

$=\frac{\partial \widetilde{P}\left(l(t), \gamma_{\mathrm{SC}}(t), a\right)}{\partial l}$,

$\frac{d \omega_{\mathrm{SC}}}{\mathrm{d} t}=-\left(\frac{\partial F}{\partial Y}+\omega \frac{\partial F}{\partial S}\right)=0$.

Equation (A.8) implies that the parameter $t$ corresponds to the rapidity $Y$. From (A.11) we can see that $\omega_{\mathrm{SC}}$ is conserved on characteristic lines, and it can be fixed from the asymptotic conditions Eq. (12) as

$$
\omega_{\mathrm{SC}}=\chi\left(\gamma_{\mathrm{SC}}\right)+\widetilde{P}\left(l(t), \gamma_{\mathrm{SC}}(t), a\left(\gamma_{\infty}\right)\right)=\chi\left(\gamma_{\infty}\right) .
$$

\footnotetext{
3 We introduce a subscript index SC for the semiclassical approximation.
}

A combination of (A.7) and (A.10) allows us to eliminate the $Y$-dependence and find $\gamma_{\mathrm{SC}}$ as a function of $l$ on a trajectory,

$$
\begin{gathered}
\frac{\mathrm{d} \gamma_{\mathrm{SC}}}{\mathrm{d} l}=-\frac{\partial \widetilde{P}\left(l(t), \gamma_{\mathrm{SC}}(t), a\right)}{\partial l} / \\
\left(\frac{\mathrm{d} \chi\left(\gamma_{\mathrm{SC}}\right)}{\mathrm{d} \gamma_{\mathrm{SC}}}+\frac{\partial \widetilde{P}\left(l(t), \gamma_{\mathrm{SC}}(t), a\right)}{\partial \gamma_{\mathrm{SC}}}\right) .
\end{gathered}
$$

From Eq. (A.7) we can obtain the trajectory equation (30).

The lines $l(Y) \equiv l_{\mathrm{SC}}(Y)$ give the set of trajectories. The trajectory that leads to the dominant contribution to $\Psi(Y, l)$ can be found from the equation

$l=l_{\mathrm{SC}}\left(Y ; \gamma_{\infty}\right)=l_{\mathrm{SP}}\left(Y ; \gamma_{\infty}\right)$

Corresponding $\gamma_{\mathrm{SC}}\left(Y, l_{\mathrm{SC}}(Y) ; \gamma_{\infty}\right)=\gamma_{\mathrm{SP}}\left(Y, l_{\mathrm{SP}}(Y) ; \gamma_{\infty}\right)$. Finally, the Eq. (A.9) can be rewritten in the form

$$
\begin{aligned}
\frac{\mathrm{d} S}{\mathrm{~d} Y}= & \left(\gamma_{\mathrm{SC}}(Y, l)-1\right) \frac{\mathrm{d} l(Y)}{\mathrm{d} Y}+\chi\left(\gamma_{\mathrm{SC}}(Y, l)\right) \\
& +\widetilde{P}\left(l, \gamma_{\mathrm{SC}}(Y, l, a)\right) .
\end{aligned}
$$

Comparing Eq. (A.15) with Eq. (25) one can see that the effective intercept is equal to $\omega_{\mathrm{eff}}(Y, l)=\left(S\left(Y, l_{\mathrm{SP}}(Y)\right)+\right.$ $\left.\frac{1}{2} l_{\mathrm{SP}}(Y)\right) / Y$.

\section{References}

1. E. Levin, L. Lipatov, M. Siddikov, Phys. Rev. D 89, 074002 (2014). arXiv:1401.4671 [hep-ph]

2. J. Bartels, L.N. Lipatov, K. Peters, Nucl. Phys. B 772, 103 (2007). arXiv:hep-ph/0610303

3. L.V. Gribov, E.M. Levin, M.G. Ryskin, Phys. Rep. 100, 1 (1983)

4. A.H. Mueller, J. Qiu, Nucl. Phys. B 268, 427 (1986)

5. L. McLerran, R. Venugopalan, Phys. Rev. D 49(2233), 3352 (1994)

6. L. McLerran, R. Venugopalan, Phys. Rev. D 50, 2225 (1994)

7. L. McLerran, R. Venugopalan, Phys. Rev. D 53, 458 (1996)

8. L. McLerran, R. Venugopalan, Phys. Rev. D 59, 094002 (1999)

9. Y.V. Kovchegov, E. Levin, Quantum Choromodynamics at High Energies. Cambridge Monographs on Particle Physics, Nuclear Physics and Cosmology. (Cambridge University Press, Cambridge, 2012)

10. A. Kovner, U.A. Wiedemann, Phys. Rev. D 66, 051502 (2002). arXiv:hep-ph/0112140

11. A. Kovner, U.A. Wiedemann, Phys. Rev. D 66, 034031 (2002). arXiv:hep-ph/0204277

12. A. Kovner, U.A. Wiedemann, Phys. Lett. B 551, 311 (2003). arXiv:hep-ph/0207335

13. E. Ferreiro, E. Iancu, K. Itakura, L. McLerran, Nucl. Phys. A 710, 373 (2002). arXiv:hep-ph/0206241

14. M. Froissart, Phys. Rev. 123, 1053 (1961)

15. A. Martin, Scattering Theory: Unitarity, Analitysity and Crossing. Lecture Notes in Physics (Springer, Berlin, 1969)

16. V.N. Gribov, Nucl. Phys. B 139, 1 (1978)

17. J. Serreau, M. Tissier, A. Tresmontant, Phys. Rev. D 89, 125019 (2014). arXiv:1307.6019 [hep-th]

18. J. Serreau, M. Tissier, Phys. Lett. B 712, 97 (2012). arXiv:1202.3432 [hep-th]

19. N. Vandersickel, D. Zwanziger, Phys. Rept. 520, 175 (2012). arXiv:1202.1491 [hep-th] 
20. J.M. Cornwall, Mod. Phys. Lett. A 28, 1330035 (2013). arXiv:1310.7897 [hep-ph]

21. J.A. Gracey, arXiv:1409.0455 [hep-ph]

22. P.J. Silva, D. Dudal, O. Oliveira, Spectral densities from the lattice. arXiv:1311.3643 [hep-lat]

23. P.J. Silva, O. Oliveira, D. Dudal, P. Bicudo, N. Cardoso, Many faces of the Landau gauge gluon propagator at zero and finite temperature: positivity violation, spectral density and mass scales. PoS QCD-TNT-III 040 (2013). arXiv:1401.1554 [hep-lat]

24. E.A. Kuraev, L.N. Lipatov, F.S. Fadin, Sov. Phys. JETP 45, 199 (1977)

25. Ya.Ya. Balitsky, L.N. Lipatov, Sov. J. Nucl. Phys. 28, 822 (1978)

26. L.N. Lipatov, Phys. Rep. 286, 131 (1997)

27. L.N. Lipatov, Sov. Phys. JETP 63, 904 (1986)

28. L.N. Lipatov, Zh. Eksp. Teor. Fiz. 90, 1536 (1986)

29. I. Balitsky, Nucl. Phys. B 46399 (1996). hep-ph/9509348

30. I. Balitsky, Phys. Rev. Lett. 81, 2024 (1998). hep-ph/9807434

31. I. Balitsky, Phys. Rev. D 60, 014020 (1999)

32. Y.V. Kovchegov, Phys. Rev. D 61, 074018 (2000). arXiv:hep-ph/9905214

33. I. Balitsky, Phys. Rev. D 60, 034008 (1999). arXiv:hep-ph/9901281

34. J. Jalilian Marian, A. Kovner, A. Leonidov, H. Weigert, Nucl. Phys. B 504, 415 (1997). hep-ph/9701284

35. J. Jalilian Marian, A. Kovner, A. Leonidov, H. Weigert, Phys. Rev. D 59, 014014 (1999). hep-ph/9706377

36. J. Jalilian Marian, A. Kovner, H. Weigert, Phys. Rev. D 59, 014015 (1999). hep-ph/9709432

37. A. Kovner, J.G. Milhano, Phys. Rev. D 61, 014012 (2000). hep-ph/9904420
38. A. Kovner, J.G. Milhano, H. Weigert, Phys. Rev. D 62, 114005 (2000)

39. H. Weigert, Nucl. Phys. A 703, 823 (2002)

40. E. Iancu, A. Leonidov, L. McLerran, Nucl. Phys. A 692, 583 (2001)

41. E. Iancu, A. Leonidov, L. McLerran, Phys. Lett. B 510, 133 (2001)

42. E. Ferreiro, E. Iancu, A. Leonidov, L. McLerran, Nucl. Phys. A 703, 489 (2002)

43. I. Gradstein, I. Ryzhik, Table of Integrals, Series, and Products, 5th edn. (Academic Press, London, 1994)

44. A.H. Mueller, D.N. Triantafyllopoulos, Nucl. Phys. B 640, 331 (2002). arXiv:hep-ph/0205167

45. D.N. Triantafyllopoulos, Nucl. Phys. B 648, 293 (2003). arXiv:hep-ph/0209121

46. S. Munier, R.B. Peschanski, Phys. Rev. D 70, 077503 (2004). arXiv:hep-ph/0401215

47. S. Munier, R.B. Peschanski, Phys. Rev. D 69, 034008 (2004). hep-ph/0310357

48. S. Munier, R.B. Peschanski, Phys. Rev. Lett. 91, 232001 (2003). hep-ph/0309177

49. F. John, Partial Differential Equations (Springer, New York, 1971)

50. E. Kamke, Differentialgleichungen Lösungsmethoden und Lösungen (Vieweg+Teubner Verlag, Leipzig, 1959)

51. R. Feynman, P. Hibbs, Quantum Mechanics and Path Integrals (McGraw-Hill, New York, 1965) 